\title{
Mortality risk during and after opioid substitution treatment: systematic review and meta-analysis of cohort studies
}

\author{
Luis Sordo, ${ }^{1,2,3}$ Gregorio Barrio, ${ }^{4}$ Maria J Bravo, 1,2 B Iciar Indave, ${ }^{1,2}$ Louisa Degenhardt,5,6 \\ Lucas Wiessing, ${ }^{7}$ Marica Ferri, ${ }^{7}$ Roberto Pastor-Barriuso ${ }^{1,2}$
}

${ }^{1}$ National Centre for

Epidemiology, Carlos III Institute

of Health, Madrid, Spain

${ }^{2}$ Consortium for Biomedical

Research in Epidemiology and

Public Health (CIBERESP)

Madrid, Spain

${ }^{3}$ Department of Preventive

Medicine and Public Health,

Faculty of Medicine,

Complutense University,

Madrid, Spain

${ }^{4}$ National School of Public

Health, Carlos III Institute of

Health, 28029 Madrid, Spain

${ }^{5}$ National Drug and Alcohol

Research Centre, University of

New South Wales, Sidney,

Australia

${ }^{6}$ Melbourne School of

Population and Global Health,

University of Melbourne,

Melbourne, Australia

7 Sector Best Practices, Knowledge Exchange and

Economic Issues, European

Monitoring Centre for Drugs and

Drug Addiction (EMCDDA),

Lisbon, Portugal

Correspondence to: $\mathrm{G}$ Barrio

gbarrio@isciii.es

Additional material is published online only. To view please visit

the journal online.

Cite this as: $B M J$ 2017;357:j1550

http://dx.doi.org/10.1136/bmj.j1550

Accepted: 17 March 2017

\section{ABSTRACT}

OBJECTIVE

To compare the risk for all cause and overdose mortality in people with opioid dependence during and after substitution treatment with methadone or buprenorphine and to characterise trends in risk of mortality after initiation and cessation of treatment.

DESIGN

Systematic review and meta-analysis.

DATA SOURCES

Medline, Embase, PsycINFO, and LILACS to September 2016.

\section{STUDY SELECTION}

Prospective or retrospective cohort studies in people with opioid dependence that reported deaths from all causes or overdose during follow-up periods in and out of opioid substitution treatment with methadone or buprenorphine.

\section{DATA EXTRACTION AND SYNTHESIS}

Two independent reviewers performed data extraction and assessed study quality. Mortality rates in and out of treatment were jointly combined across methadone or buprenorphine cohorts by using multivariate random effects meta-analysis.

\section{RESULTS}

There were 19 eligible cohorts, following 122885 people treated with methadone over 1.3-13.9 years and 15831 people treated with buprenorphine over 1.1-4.5 years. Pooled all cause mortality rates were 11.3 and 36.1 per 1000 person years in and out of methadone treatment (unadjusted out-to-in rate ratio 3.20, 95\% confidence interval 2.65 to 3.86 ) and reduced to 4.3 and 9.5 in and

\section{WHAT IS ALREADY KNOWN ON THIS TOPIC}

Opioid substitution treatment is effective in suppressing illicit opioid use and reducing all cause and overdose mortality

Growing evidence suggests that mortality during and after opioid substitution treatment is time varying and differs by type of drug

\section{WHAT THIS STUDY ADDS}

In patients using methadone maintenance treatment there are, on average, 25 fewer deaths $/ 1000$ person years than in patients who discontinue it. Mortality risk among opioid users during treatment is less than a third of that expected in the absence of opioid substitution treatment

Buprenorphine maintenance treatment is probably also effective in reducing mortality in opioid users, but quantification of averted deaths requires further studies

The mortality risk in the induction phase of methadone (first four weeks) is high but seems to decreases substantially during this period, with a further stabilisation at around six deaths/1000 person years in the remaining time in treatment. This did not occur with buprenorphine. The mortality risk in the four weeks immediately after cessation of either treatment is high and could exceed 30 deaths/1000 person years out of buprenorphine treatment $(2.20,1.34$ to 3.61$)$. In pooled trend analysis, all cause mortality dropped sharply over the first four weeks of methadone treatment and decreased gradually two weeks after leaving treatment. All cause mortality remained stable during induction and remaining time on buprenorphine treatment. Overdose mortality evolved similarly, with pooled overdose mortality rates of 2.6 and 12.7 per 1000 person years in and out of methadone treatment (unadjusted out-to-in rate ratio 4.80, 2.90 to 7.96 ) and 1.4 and 4.6 in and out of buprenorphine treatment.

\section{CONCLUSIONS}

Retention in methadone and buprenorphine treatment is associated with substantial reductions in the risk for all cause and overdose mortality in people dependent on opioids. The induction phase onto methadone treatment and the time immediately after leaving treatment with both drugs are periods of particularly increased mortality risk, which should be dealt with by both public health and clinical strategies to mitigate such risk. These findings are potentially important, but further research must be conducted to properly account for potential confounding and selection bias in comparisons of mortality risk between opioid substitution treatments, as well as throughout periods in and out of each treatment.

\section{Introduction}

Opioid dependence is a rising drug use disorder with substantial contribution to the global disease burden. The absolute number (age standardised prevalence) of people with opioid dependence worldwide increased from 10.4 million $(0.20 \%)$ in 1990 to 15.5 million $(0.22 \%)$ in 2010, and the disability adjusted years of life lost attributable to opioid dependence rose from 5.3 million (0.21\% of global disease burden) in 1990 to 9.2 million $(0.37 \%)$ in $2010 .^{1}$

Opioid substitution treatment, either with methadone or buprenorphine, has been shown to be safe and effective in suppressing illicit opioid use, ${ }^{23}$ improving physical and mental wellbeing, ${ }^{45}$ and reducing all cause and overdose mortality. ${ }^{6}$ Growing evidence, however, suggests that mortality experience during and after opioid substitution treatment is time varying and differs by type of drug. ${ }^{6-10}$ Methadone, a full opioid agonist, might pose an excess risk of death from overdose during treatment induction if initial doses are too high or coexist with illicit opioid use. ${ }^{6710}$ Buprenorphine, a partial agonist, is less effective in retaining patients in treatment, ${ }^{3}$ which seems a major weakness because mortality increases significantly in the period immediately after treatment stops. ${ }^{10}{ }^{11} \mathrm{Nev}-$ ertheless, studies of individual cohorts have generally 
been under-powered to detect potential differences in mortality risk by time interval in and out of treatment, and long term large trials comparing the effect of methadone and buprenorphine on all cause and overdose mortality are hardly feasible. ${ }^{3}$ In this context, a systematic review of cohort studies can provide valuable evidence on the mortality experience of opioid dependent people at different periods of methadone and buprenorphine substitution treatment that could guide clinicians and policymakers in the optimal provision of treatment. ${ }^{12}$

In this systematic review and meta-analysis we synthesised results from cohort studies on mortality among patients receiving opioid substitution treatment to obtain pooled estimates of all cause and overdose mortality rates during periods in and out of treatment with methadone and buprenorphine, evaluate heterogeneity of mortality rates across cohort characteristics, and investigate changes in mortality rates over time in and out of treatment, particularly within the first weeks after treatment initiation and cessation.

\section{Methods}

\section{Search strategy and inclusion criteria}

We searched Medline, Embase, PsycINFO, and LILACS by using different combinations of free text and database specific index terms related to the topics of opioid dependence, opioid substitution treatment, mortality, and cohort studies. Details of the full search strategy are provided in appendix 1 . The search was updated to September 2016, with no language restrictions. We also searched the Cochrane Database of Systematic Reviews, reviewed the reference lists of relevant original papers and reviews, screened articles in the PubMed "related citations" section, searched online for technical reports and monographs, and consulted experts and investigators with ongoing studies in opioid dependence. We followed the PRISMA statement for reporting systematic reviews and meta-analyses. ${ }^{13}$

We included cohort studies comparing mortality among people with opioid dependence. To be eligible, studies had to include follow-up data during and after opioid substitution treatment with methadone or buprenorphine. The prespecified exclusion criteria were as follows:

- No data in humans

- No original research (reviews, editorials, non-research letters, protocols)

- Study design other than observational cohort (clinical trials, case-control studies, cross sectional surveys, case reports, case series, qualitative research)

- Study not focused on people with opioid dependence

- People in prison or recently released

- Treatment unknown or other than methadone or buprenorphine maintenance (methadone detoxification, opioid antagonist therapy, therapeutic community)

- No all cause or overdose mortality as outcome or no deaths over follow-up

- Insufficient data to compute mortality rates during periods in and out of treatment (number of deaths and person years broken down by follow-up period in and out of treatment).

For cohorts originating several reports, we used data from non-overlapping follow-up periods from each report whenever possible or selected the publication with the longest follow-up.

\section{Study selection and data extraction}

Two investigators independently reviewed the titles and abstracts identified in the search and retrieved articles to determine eligibility and to extract study data. Disagreements or uncertainties were resolved by consensus with an additional investigator.

For each eligible study, we retrieved information on baseline population characteristics, including study location, sex and age distribution, primary opioid of misuse, and prevalence of opioid injection, non-opioid drug use, HIV infection, and psychiatric and medical comorbidity; number of cohort participants entering opioid substitution treatment during the study period (untreated participants and those under other types of treatment were excluded); treatment features, including drug type (methadone or buprenorphine), average daily dose, induction method (inpatient or ambulatory), and provider (addiction medicine specialist or general practitioner); and main follow-up characteristics, including calendar period, average length of follow-up from the start of maintenance treatment (excluding any previous detoxification period), loss to follow-up, and mortality outcomes. We also registered detailed information on the number of deaths, person years at risk, and mortality rates from all causes and overdose during follow-up periods in and out of treatment and, whenever possible, during specific time intervals since treatment initiation and cessation. Finally, we registered information on first and subsequent treatment episodes (table A in appendix 2) and on completeness of treatment (table B in appendix 2).

We specifically designed a quality assessment form based on standardised and extensively used instruments: the methodology checklist for cohort studies developed by the Scottish Intercollegiate Guidelines Network ${ }^{14}$ and the checklist for drug related studies developed by the National Drug and Alcohol Research Centre, Australia. ${ }^{15}$ The design process, based on a thorough review of the above sources, included the development of different proposals, discussion of their appropriateness, and final agreement among the authors. The final version comprised separate sections according to the study design and was based on a "star system" score approach, ${ }^{16}$ including a general appraisal of external and internal validity and of the biases relevant to cohort studies, plus an ad hoc assessment of reporting for studies on mortality during and after opioid substitution treatment (appendix 3).

\section{Statistical analysis}

For each selected cohort, we calculated crude mortality rates from all causes and overdose during periods in 
and out of treatment by dividing the number of deaths registered in each period by the person years contributed by all participants to that period. If not explicitly reported, we derived the number of person years by treatment period from the available summary statistics on follow-up and treatment retention or the reported mortality rates. We computed exact 95\% confidence intervals for the underlying mortality rates by assuming a Poisson distribution for the observed number of deaths and fixed person years at risk.

Cause specific mortality rates in and out of treatment were jointly combined across all methadone or buprenorphine cohorts by using a bivariate random effects meta-analysis on log transformed mortality rates in both treatment periods. ${ }^{17-19}$ This bivariate meta-analytic approach allowed estimation of not only the pooled mortality rates and 95\% confidence intervals in and out of treatment but also the pooled rate difference and rate ratio comparing periods out of and in treatment, with confidence intervals that took into account the correlation between rates in both treatment periods.

To evaluate whether cause specific mortality rates differed by baseline population characteristics, treatment delivery, and follow-up features of methadone cohorts, we included the location (Europe/Israel, North America, or Australia), prevalence of opioid injection $(<100$ or $100 \%)$, percentage of men $(<75$ or $\geq 75 \%)$, mean age $(<35$ or $\geq 35)$, average methadone dose $(\leq 80$ or $>80$ $\mathrm{mg} /$ day), percentage of inpatient induction ( 0 or $>0 \%$ ), treatment provider (specialist or general practitioner/ mixed), midpoint follow-up period ( $<2000$ or $\geq 2000$ ), and percentage loss to follow-up ( $<10$ or $\geq 10 \%)$ in each methadone cohort as single predictors in separate bivariate random effects meta-regression models on log transformed mortality rates in and out of treatment. ${ }^{17} 18$ We contrasted heterogeneity of pooled mortality rates by these cohort characteristics through likelihood ratio tests comparing nested meta-regression models with and without the predictor fitted through maximum likelihood. The limited number of buprenorphine cohorts precluded a similar heterogeneity analysis across these cohorts.

For methadone or buprenorphine cohorts that reported mortality data by time interval in and out of treatment, we combined cause specific mortality rates before and after four weeks of treatment initiation and cessation through a multivariate random effects meta-analysis on log transformed mortality rates in these time-by-treatment intervals. To obtain pooled trends in all cause mortality risk over time in and out of methadone substitution treatment, we fitted a bivariate random effects meta-regression of log transformed rates on a quadratic linear spline function of log time with knot at four weeks. ${ }^{20}$ This quadratic linear spline allowed for a quadratic trend in the first four weeks in and out of treatment and forced the trend to be linear thereafter to avoid implausible shapes at long follow-up times. The resulting pooled trends were virtually insensitive to different knot locations at three, four, or five weeks.
We examined the overall heterogeneity in mortality rates across studies, as well as the residual heterogeneity beyond that explained by study level characteristics, with the multivariate extension of the Cochran $\chi^{2}$ test and quantified it with the extended $\mathrm{I}^{2}$ statistic, ${ }^{1821}$ which described the proportion of total variation in study specific mortality rates because of heterogeneity. The influence of each study on pooled estimates was evaluated by removal of each individual study from the analysis. Publication bias and genuine small study effects were assessed with the extended Egger test, allowing for heterogeneity. 2223

In all the above multivariate random effects meta-analytic models, we assumed the correlations within cohorts in the estimated mortality rates to be zero as they were obtained over different follow-up periods in and out of treatment. Nevertheless, we performed sensitivity analyses assuming positive within cohort correlations between mortality rates in and out of treatment of $0.25,0.50,0.75$, and 0.95 for all studies, and the pooled rates and their standard errors were virtually identical to those obtained under uncorrelated rates within cohorts (changes of $-1.3 \%$ to $1.6 \%$ in pooled rates and $-5.7 \%$ to $7.6 \%$ in standard errors). All models were fitted through restricted maximum likelihood methods with unstructured between cohort covariance matrix with the R package mvmeta ( $R$ Foundation for Statistical Computing). The estimated between cohort correlations in the underlying rates in and out of treatment were 0.93 for all cause mortality and 0.81 for overdose mortality, with no estimation problems in any model regarding between cohort correlations at the boundary of the parameter space of 1 .

\section{Patient involvement}

No patients were involved in setting the research question or outcome measures, nor were they involved in developing plans for design or implementation of the study. No patients were asked to advise on interpretation or writing up on results. There are no plans to disseminate the results of the research to study participants or the relevant patient community.

\section{Results}

\section{Study characteristics}

Of the 2033 distinct records identified through the search, we selected 19 cohort studies reported in 20 articles $^{6-1024-38}$ (two articles ${ }^{27} 28$ reported different mortality outcomes from the same cohort) that met the inclusion criteria and provided mortality data in people with opioid dependence during and after opioid substitution treatment (fig 1). Tables 1 and 2 show the main characteristics of selected cohorts. The studies were published between 1974 and 2016 and were all carried out in high income countries: 11 in Europe/Israel, four in North America, and four in Australia. There were mixed baseline prevalences of opioid injection and HIV infection, except four cohorts that enrolled only opioid injectors, two of them further restricted to patients positive for HIV. All but four cohorts included patients with concurrent use of non-opioid drugs, with limited information 
Distinct records identified $(n=2033)$ :

Medline $(n=1215)$

Embase $(n=729) \quad$ LILACS $(n=182)$

PsycINFO $(n=486) \quad$ Other searches $(n=102)$

Records excluded after title and abstract review $(n=1705)$ :

No data in humans $(\mathrm{n}=120)$

$\rightarrow$ No original research $(n=126)$

Design other than observational cohort $(n=462)$

Not focused on opioid dependent people $(n=997)$

Full text articles retrieved and assessed for eligibility $(n=328)$

Articles excluded $(n=308)$ :

Imprisoned or recently released population $(n=22)$

Treatment unknown or other than methadone or

buprenorphine maintenance $(n=133)$

No all cause or overdose mortality as outcome $(n=44)$

Insufficient data to compute mortality rates during

follow-up periods in and out of treatment $(n=72)$

Secondary publication $(n=37)$

Articles included in meta-analysis $(n=20)$

Fig 1 | Selection process of cohort studies on mortality among people receiving opioid substitution treatment

on psychiatric and medical comorbidities. More than $70 \%$ of participants were men, and the mean age at baseline ranged from 23.4 to 39.6 (table 1).

Methadone was prescribed in 18 cohorts including 122885 participants over the period 1965-2010, and buprenorphine was used in three cohorts including 15831 participants over 1990-2010 (table 2). The average daily dose ranged from $47 \mathrm{mg}$ to $116 \mathrm{mg}$ of methadone and $10 \mathrm{mg}$ to $12 \mathrm{mg}$ of buprenorphine. Treatment was initiated on an ambulatory basis in all recent cohorts and provided by addiction medicine specialists in 11 cohorts and general practitioners or mixed staff in eight cohorts. The average follow-up after initiation varied between 1.3 and 13.9 years for methadone and 1.1 and 4.5 years for buprenorphine, with loss to follow-up exceeding $10 \%$ in only four cohorts. All cause mortality during follow-up periods in and out of treatment was reported in all but two methadone cohorts and overdose mortality in 11 methadone cohorts and one buprenorphine cohort. Most studies were of moderate quality. They ranged from 3 to 13 points on a 16 point scale, with a median of 8 points.

\section{All cause mortality during and after opioid substitution treatment}

All cause mortality rates varied widely across the 16 methadone cohorts (overall $\mathrm{I}^{2}=98 \%, \mathrm{P}<0.001$ ), although rates were consistently higher out of treatment than in treatment (fig 2). The pooled all cause mortality rates were 11.3 and 36.1 deaths per 1000 person years in and out of methadone treatment, respectively, with an unadjusted pooled rate difference for periods out and in treatment of 24.9 deaths (95\% confidence interval 13.6 to 36.1) per 1000 person years and an unadjusted pooled rate ratio of 3.20 (2.65 to 3.86). In heterogeneity analyses (table 3 ), the pooled all cause mortality rates in and out of methadone treatment were significantly higher in studies that mostly enrolled opioid injectors who were positive for HIV than in studies including both injectors and non-injectors, and marginally higher in studies conducted in Europe and North America than in Australia.

The pooled all cause mortality rates in the three buprenorphine cohorts were 4.3 and 9.5 deaths per 1000 person years in and out of treatment, respectively (fig 2). The unadjusted pooled rate difference for periods out and in treatment was 5.2 deaths (95\% confidence interval -1.0 to 11.4 ) per 1000 person years and the unadjusted pooled rate ratio was 2.20 (1.34 to 3.61).

After we removed each individual cohort, the pooled all cause mortality rates ranged from 10.6 to 11.9 deaths per 1000 person years in methadone treatment and from 32.5 to 39.1 deaths per 1000 person years out of methadone treatment. With buprenorphine treatment these figures were 3.3 to 5.6 deaths and 7.7 to 12.3 deaths per 1000 person years, respectively. There was some evidence of small study effects on all cause mortality $(\mathrm{P}=0.05)$, with higher rates in small cohorts that mostly enrolled opioid injectors who were positive for HIV (table 1 and fig 2).

Five methadone cohorts and one buprenorphine cohort reported all cause mortality rate ratios for periods out of and in treatment, with various degrees of adjustment for patient demographics, severity of drug use, treatment delivery, and comorbidity (table 4). After adjustment, out-to-in rate ratios remained unchanged in two methadone cohorts and increased by $15.5-35.5 \%$ in the other three methadone cohorts and by $16.8 \%$ in the buprenorphine cohort.

\section{Overdose mortality during and after opioid substitution treatment}

The pooled overdose mortality rates across the 11 methadone cohorts were 2.6 and 12.7 overdose deaths per 1000 person years in and out of methadone treatment, respectively (fig 3), with an unadjusted pooled rate difference comparing periods out and in treatment of 10.0 overdose deaths ( $95 \%$ confidence interval 2.6 to 17.4) per 1000 person years and an unadjusted pooled rate ratio of 4.80 (2.90 to 7.96 ). There was moderate heterogeneity between studies in mortality rates in treatment $\left(\mathrm{I}^{2}=66 \%\right.$, $\mathrm{P}=0.001)$ and strong heterogeneity in rates out of treatment $\left(\mathrm{I}^{2}=97 \%, \mathrm{P}<0.001\right)$, with significantly higher rates out of treatment among methadone patients in specialist services than in primary care (table 3 ). In the single buprenorphine cohort there were 1.4 and 4.6 fatal overdoses per 1000 person years in and out of treatment (fig 3).

After we removed each methadone cohort, the pooled overdose mortality rates varied between 2.4 and 2.8 fatal overdoses per 1000 person years in methadone treatment and between 10.6 and 14.9 fatal overdoses per 1000 person years out of treatment. There was no evidence of publication bias or other small study effects on overdose mortality $(\mathrm{P}=0.14)$. Adjustment for potential confounders did not produce any change in overdose mortality rate ratios for periods out compared with in treatment in three methadone cohorts, but resulted in $25.8 \%$ and $28.6 \%$ larger rate ratios in two other methadone cohorts (table 4). 


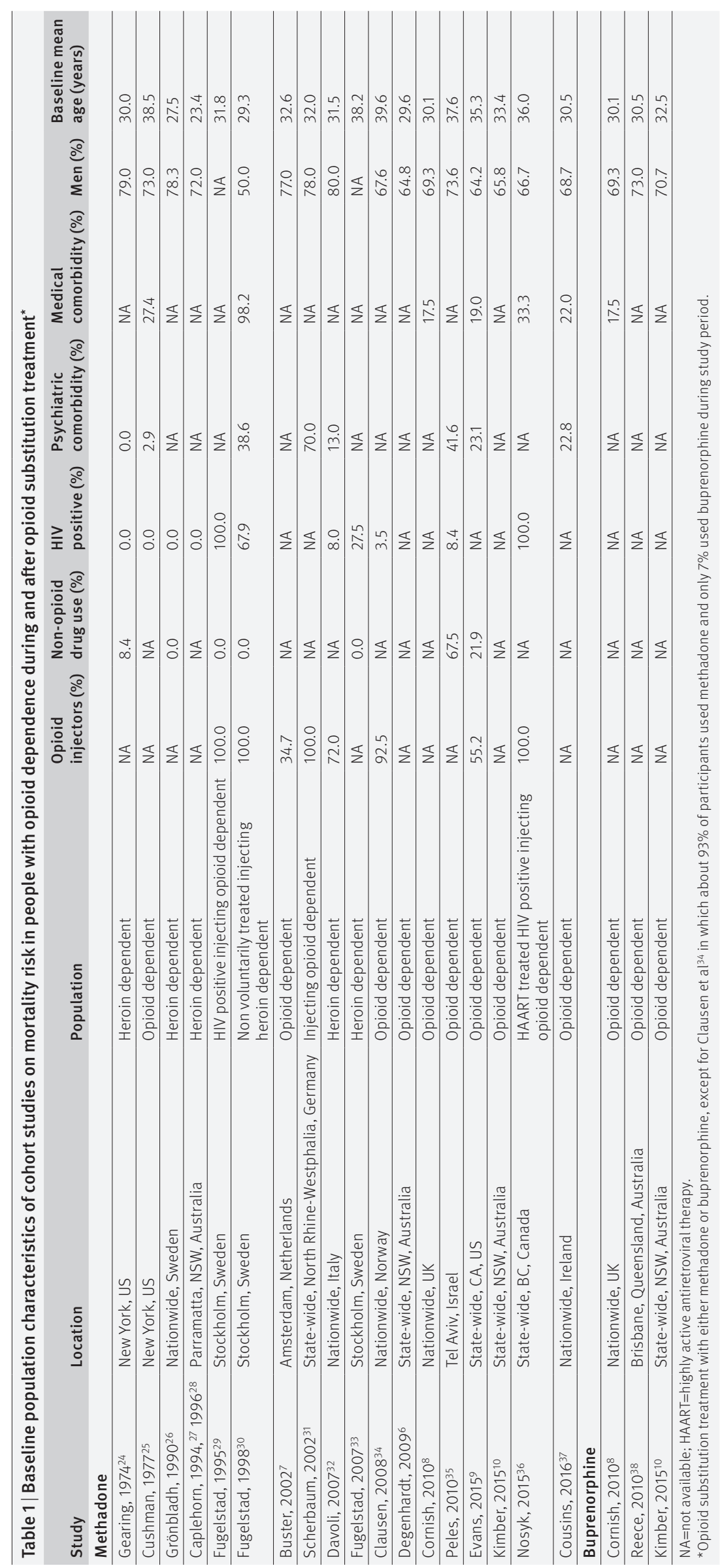

\section{All cause mortality by time interval in and out of treatment}

Six recent methadone cohorts provided all cause mortality data disaggregated by time interval in and out of treatment (fig 4). After we excluded a selective high risk cohort of injectors who were HIV positive and received highly active antiretroviral therapy, the pooled all cause mortality rates were 11.4 and 5.8 deaths per 1000 person years in the first four weeks and the remaining time on methadone treatment, respectively, with an unadjusted pooled rate difference for initial compared with subsequent treatment periods of 5.6 deaths (95\% confidence interval -2.4 to 13.6) per 1000 person years and an unadjusted pooled rate ratio of 1.97 (0.94 to 4.10). Similarly, the pooled all cause mortality dropped from 32.1 deaths per 1000 person years in the first four weeks after stopping methadone treatment to 13.5 deaths per 1000 person years thereafter, resulting in an unadjusted pooled rate difference for initial compared with subsequent periods out of treatment of 18.6 deaths (2.9 to 34.3) per 1000 person years and an unadjusted pooled rate ratio of 2.38 (1.51 to 3.74).

In continuous time trend analysis (fig 5), there were significant departures from linearity in trends in all cause mortality risk over time in and out of methadone treatment $(\mathrm{P}=0.04)$. The pooled all cause mortality rates decreased sharply from 37.4 to 6.4 deaths per 1000 person years throughout the initial four weeks of methadone treatment and were fairly stable thereafter. In contrast, after people left methadone treatment, the pooled all cause mortality remained high at between 24.1 and 35.2 deaths per 1000 person years during the initial two weeks out of treatment and decreased progressively afterwards.

In the two buprenorphine cohorts reporting disaggregated data, the pooled all cause mortality remained equally low at 4.5 deaths per 1000 person years during and after the first four weeks of buprenorphine treatment (fig 4). The pooled all cause mortality, however, increased to 32.0 deaths per 1000 person years in the first four weeks after people stopped buprenorphine treatment and reduced thereafter to 10.9 deaths per 1000 person years, with an unadjusted pooled rate difference for initial compared with subsequent periods out of treatment of 21.2 deaths ( $95 \%$ confidence interval -5.9 to 48.2 ) per 1000 person years and an unadjusted pooled rate ratio of 2.94 (1.34 to 6.46).

\section{Overdose mortality by time interval in and out of treatment}

Only three methadone cohorts and one buprenorphine cohort reported overdose mortality by time interval (fig 6). The pooled overdose mortality rates were 3.5 and 2.0 overdose deaths per 1000 person years in the first four weeks and the rest of time on methadone treatment and 4.2 and 3.4 overdose deaths per 1000 person years during and after the first four weeks out of treatment. In the single buprenorphine cohort, there were 0.9 and 1.5 fatal overdoses per 1000 person years before and after the first four weeks on buprenorphine treatment, and 10.8 and 4.2 fatal overdoses per 1000 person years before and after four weeks out of treatment, respectively. 


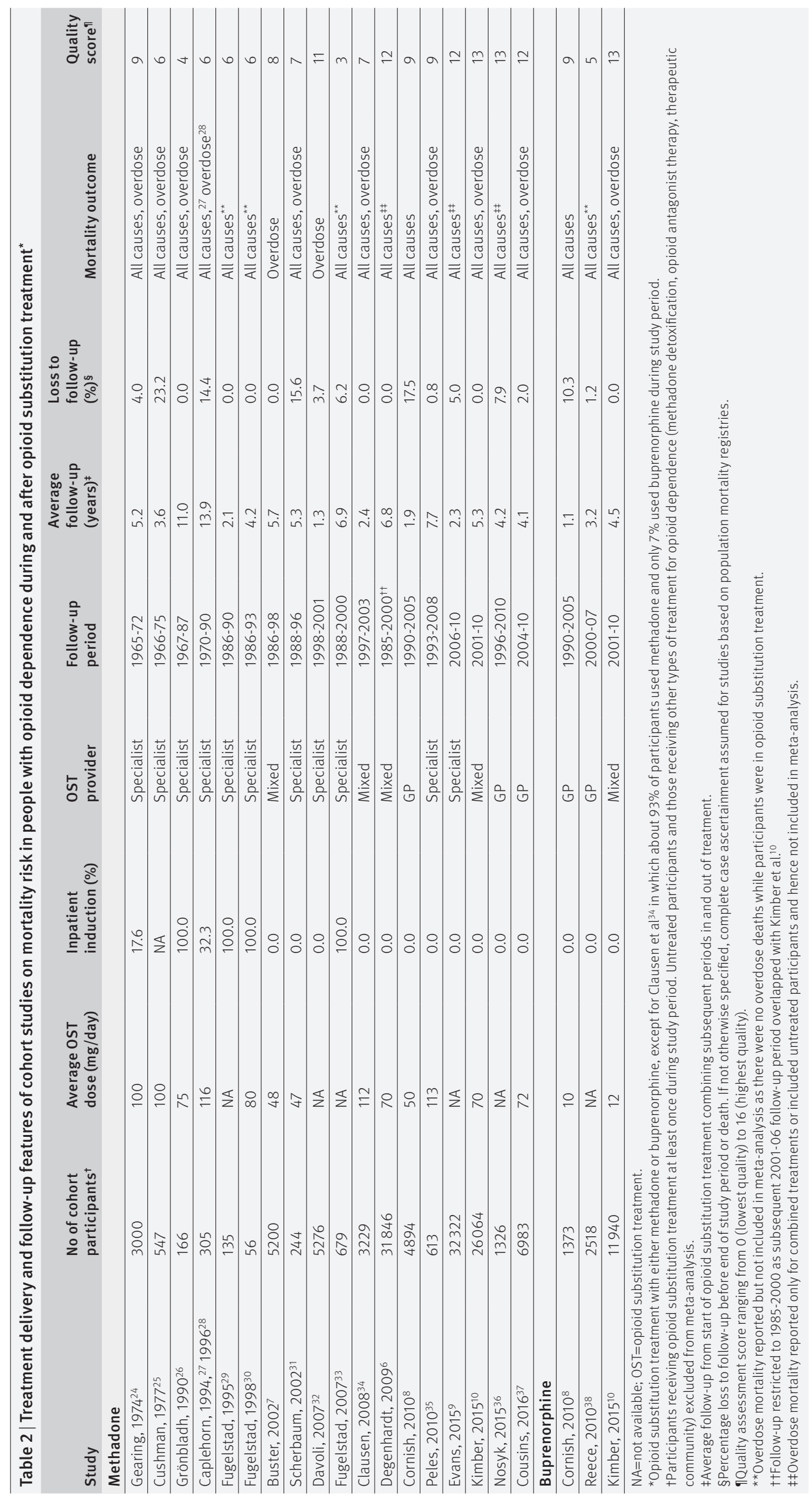




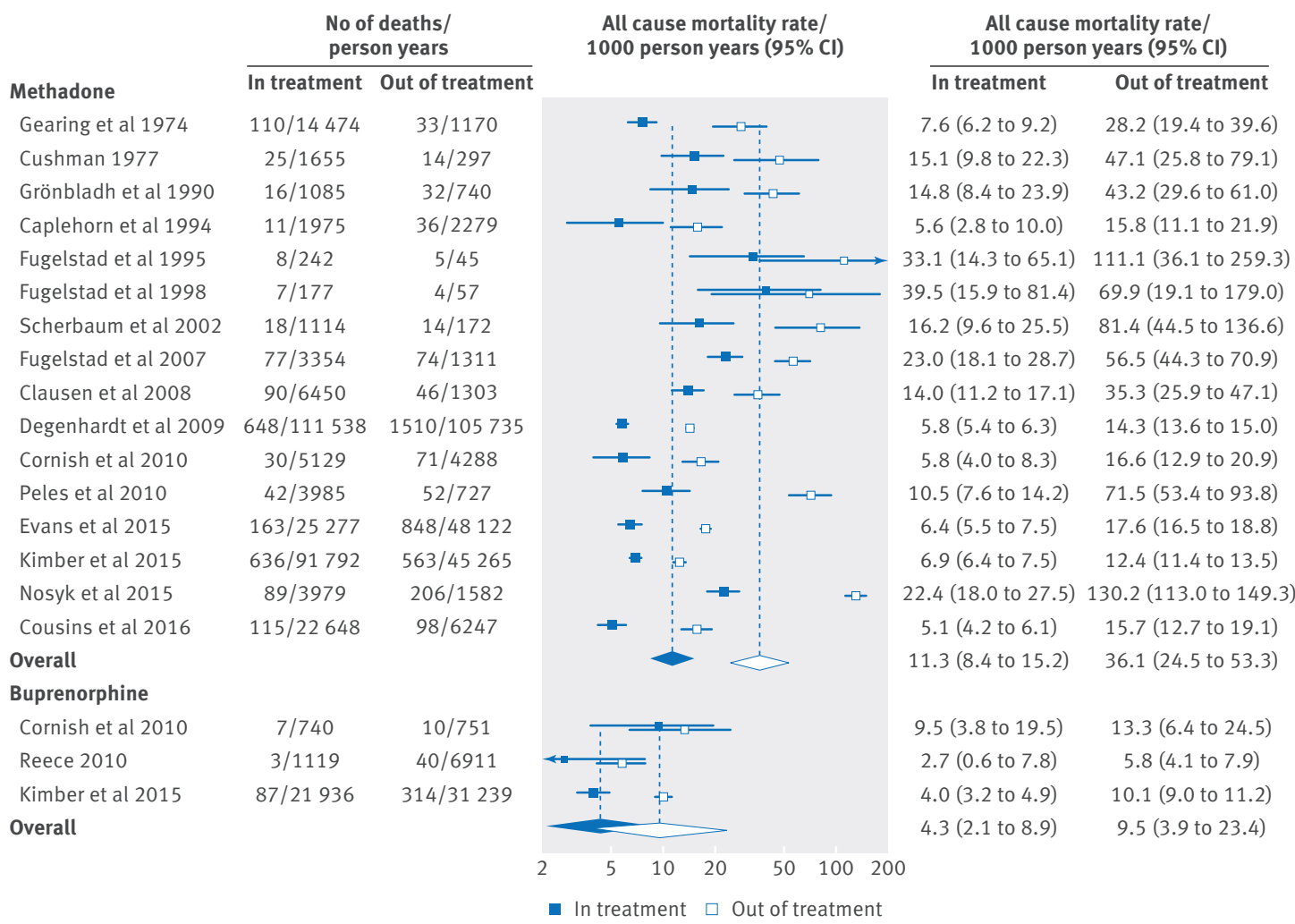

Fig 2 | All cause mortality rates in and out of opioid substitution treatment with methadone or buprenorphine and overall pooled all cause mortality rates, 1974-2016. Area of each square is proportional to study weight in meta-analysis. Horizontal lines represent exact $95 \%$ confidence intervals based on Poisson distribution. Diamonds represent pooled all cause mortality rates during periods in and out of treatment across all methadone or buprenorphine cohorts estimated from bivariate random effects meta-analysis on log transformed rates in both treatment periods

\section{Discussion}

\section{Main findings}

Our review suggests that time spent in opioid substitution treatment with methadone is associated with an average reduction of 25 deaths/1000 person years ( $95 \%$ confidence interval 14 to 36). The rate in treatment was less than a third of the rate out of treatment, with the greatest difference in deaths from overdose. The all cause mortality risk during treatment is much higher in the first four weeks than in the remainder of treatment. Mortality risk is also higher in the first four weeks after cessation than in the remainder time out of treatment.

Based on three cohorts (two from Australia), findings also suggest that opioid substitution treatment with buprenorphine could be associated with a reduction in mortality, with a similar risk across all time in treatment (about four deaths/1000 person years) consistent with its safety profile, ${ }^{39-44}$ and a risk after cessation higher in the first four weeks than in the remainder of time out of treatment (32 versus 11 deaths/1000 person years).

\section{Mortality risk during specific periods in and out of methadone treatment}

The larger relative effect size (as measured by rate ratio) for overdose than non-overdose deaths with methadone substitution ${ }^{6113445}$ is consistent with previous findings, although the effect on non-overdose deaths had not been previously reviewed. This disparity is consistent with what would be expected given methadone's effect on reducing use of heroin and other illicit opioids. $^{3234}$

Our finding that the first four weeks after onset (11.4 deaths/1000 person years) and cessation (32.1/1000 person years) of methadone treatment are the highest risk periods suggests that these are key periods during which to focus efforts for prevention of drug related deaths. Moreover, within periods of methadone treatment there is a swift decline in risk from first to fourth week, followed by a stable trend, whereas after cessation there is a high but quite stable risk during the first four weeks and a progressive decline afterward. This is important because some patients cycle in and out of opioid substitution treatment $\mathrm{t}^{46-50}$ and are therefore exposed to repeated periods of high risk for mortality. Such changes probably mainly reflect changes in risk of fatal overdose, ${ }^{6} 32$ but this has not been accurately assessed because only two cohorts reported overdose data specifically. ${ }^{710}$ The increased risk during the first few weeks of treatment could be explained by an accumulation of methadone that exceeds the opioid tolerance level (the mean elimination half life for methadone is 22 hours), ${ }^{51}$ as opioid tolerance does not seem to fully protect against respiratory depression. ${ }^{52}$ Psychological factors and concomitant use of other respiratory depressant drugs or cocaine could also play a role. ${ }^{78405354}$ 


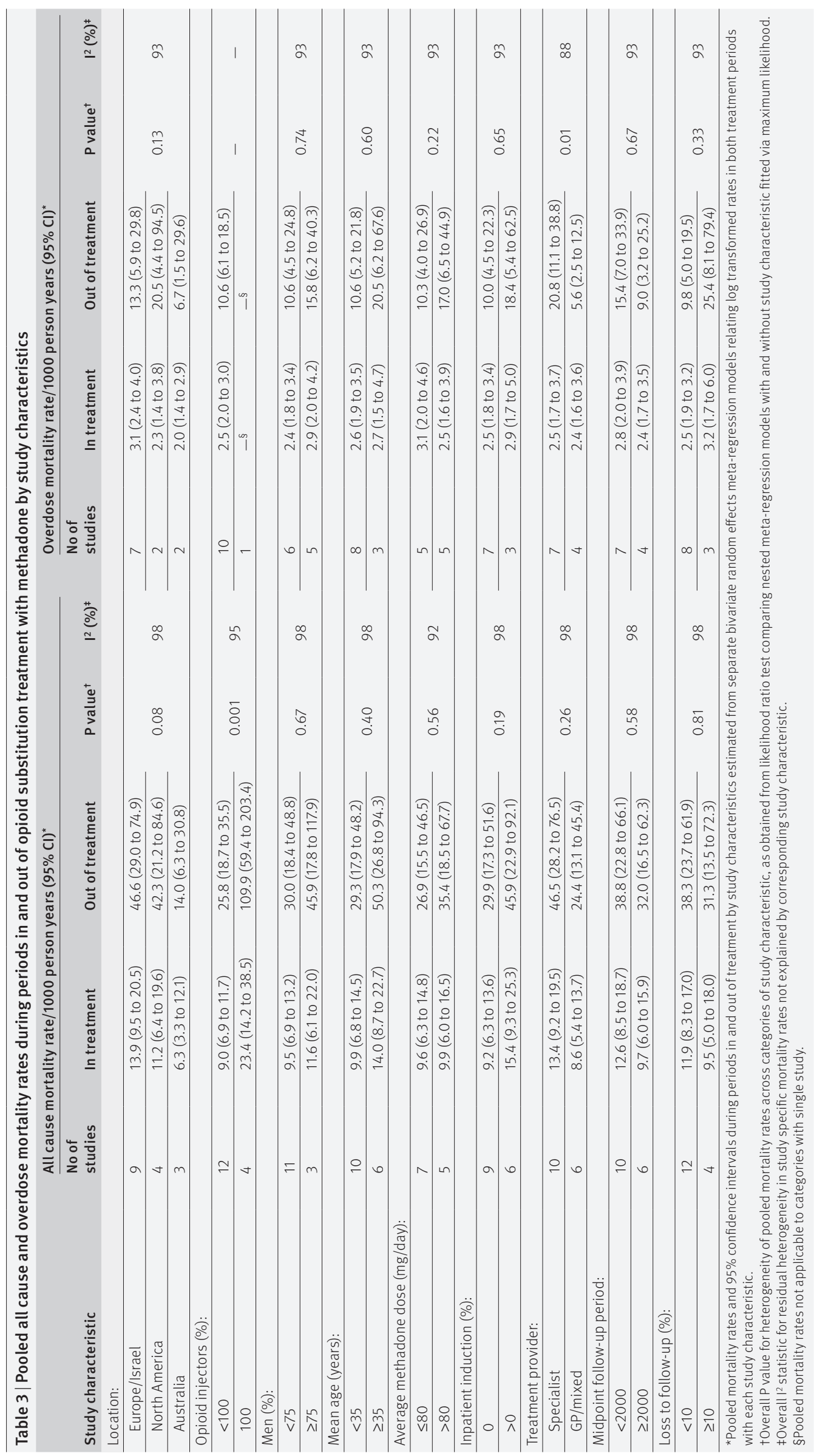

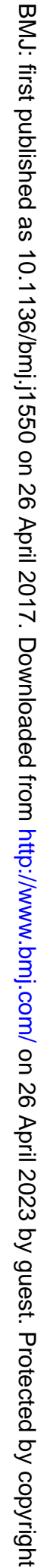




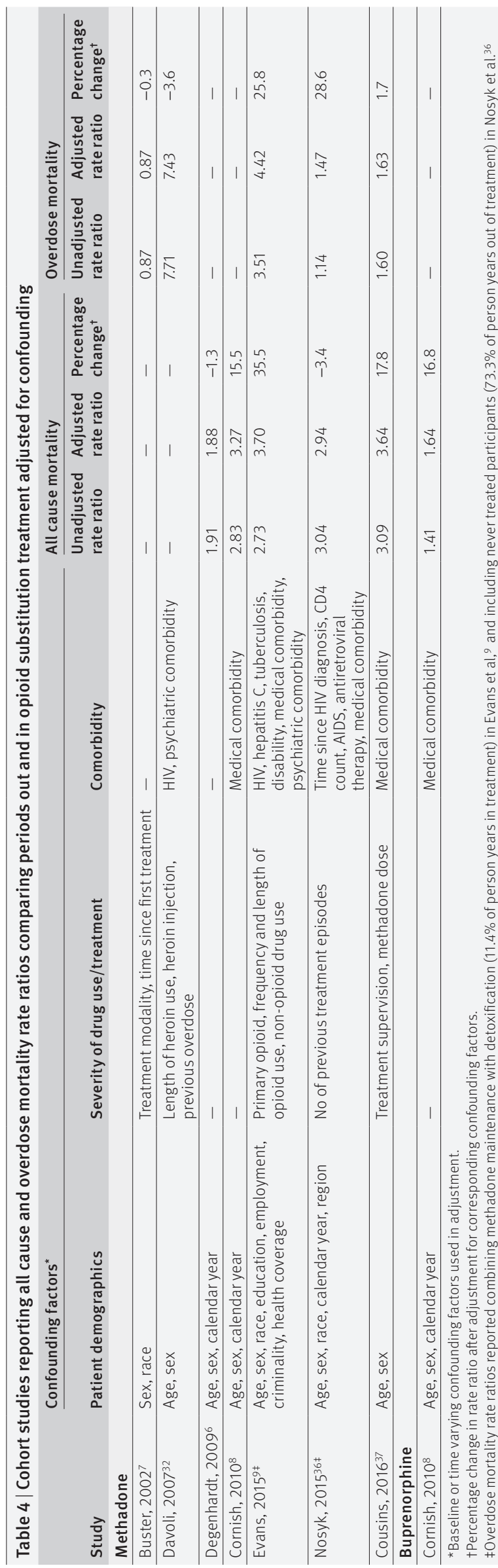

Although the long half life of methadone could contribute to the increase in mortality in the first three to four days after cessation of treatment, there was a large increase in mortality across the first four weeks after cessation compared with the treatment period. Such increased mortality could be explained by loss of tolerance to the toxic effects of heroin, assuming that many patients return to use, ${ }^{6832475556}$ and probably also by the use of multiple legal and illicit substances during that period. Similar peaks in drug related mortality occur in other situations where opioid tolerance is probably diminished (for example, after prison release or hospital discharge ${ }^{57-59}$ ). Lifestyle factors and comorbid issues such as mental health problems could also increase mortality from other external causes, as deaths from suicide and injury are also more common during that period..$^{53}$

\section{Mortality risk during specific periods in and out of buprenorphine treatment}

Data from three cohorts (two from Australia) suggest that substitution treatment with buprenorphine could reduce all cause mortality, although the disparity in mortality rates in periods in and out of treatment did not reach significance. Findings also suggest a significantly increased mortality in the first four weeks after cessation of treatment compared with the remaining time out of treatment (32.0 versus 10.9/1000 person years), while during the treatment period there was no difference between the first four weeks and the remaining time in treatment. It is difficult to draw firm conclusions because of limited evidence from similar settings: $96 \%$ of included deaths and $98 \%$ of person years came from Australian studies (with 87\% and 86\% from the study by Kimber and colleagues ${ }^{13}$ ).

\section{Comparative effectiveness of buprenorphine and methadone substitution treatment}

Other sources of data have suggested that buprenorphine could be more effective than methadone in reducing mortality, especially from overdose. ${ }^{39} 4243$ Unlike methadone, there is a ceiling for respiratory depressant effects of buprenorphine as dose increases, and the probability of triggering arrhythmias is lower for buprenorphine than methadone. ${ }^{414460}$ When we compared the mortality between periods in and out of treatment, first within those taking methadone and then within those taking buprenorphine, we found a greater reduction in mortality with methadone than with buprenorphine (measured as the rate difference or rate ratio between periods). When we compared mortality between them, first within the treatment period and then in the period off treatment, we found a significantly lower mortality with buprenorphine than methadone in both periods.

Our conclusions must remain tentative until further studies in varied treatment settings and contexts are undertaken to examine this issue. Differences in mortality might also reflect confounding through differences in characteristics of patients (such as age, severity of opioid dependence, injecting drug use, other 


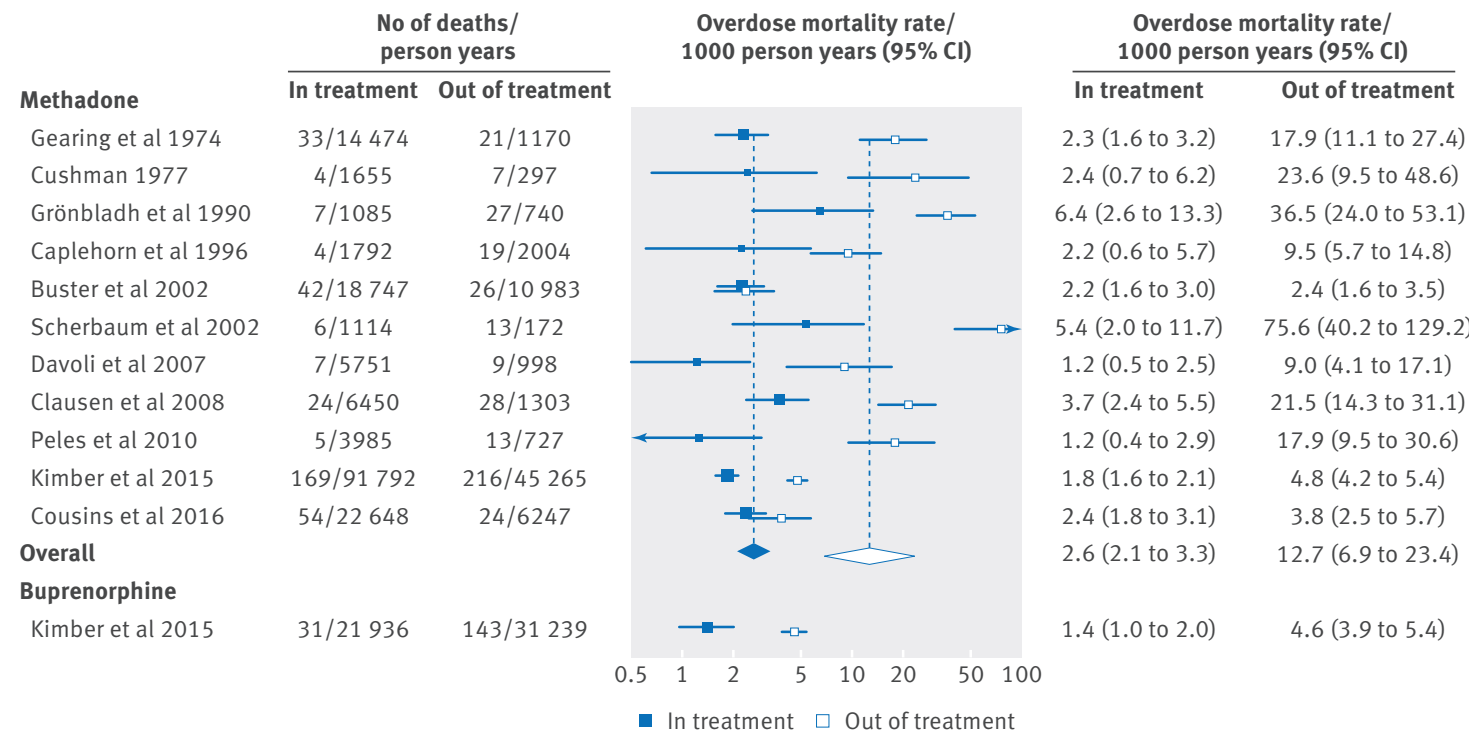

Fig 3 | Overdose mortality rates in and out of opioid substitution treatment with methadone or buprenorphine and overall pooled overdose mortality rates, 1974-2016. Area of each square is proportional to study weight in meta-analysis. Horizontal lines represent exact $95 \%$ confidence intervals based on Poisson distribution. Diamonds represent pooled overdose mortality rates during periods in and out of treatment across all methadone cohorts estimated from bivariate random effects meta-analysis on log transformed rates in both treatment periods

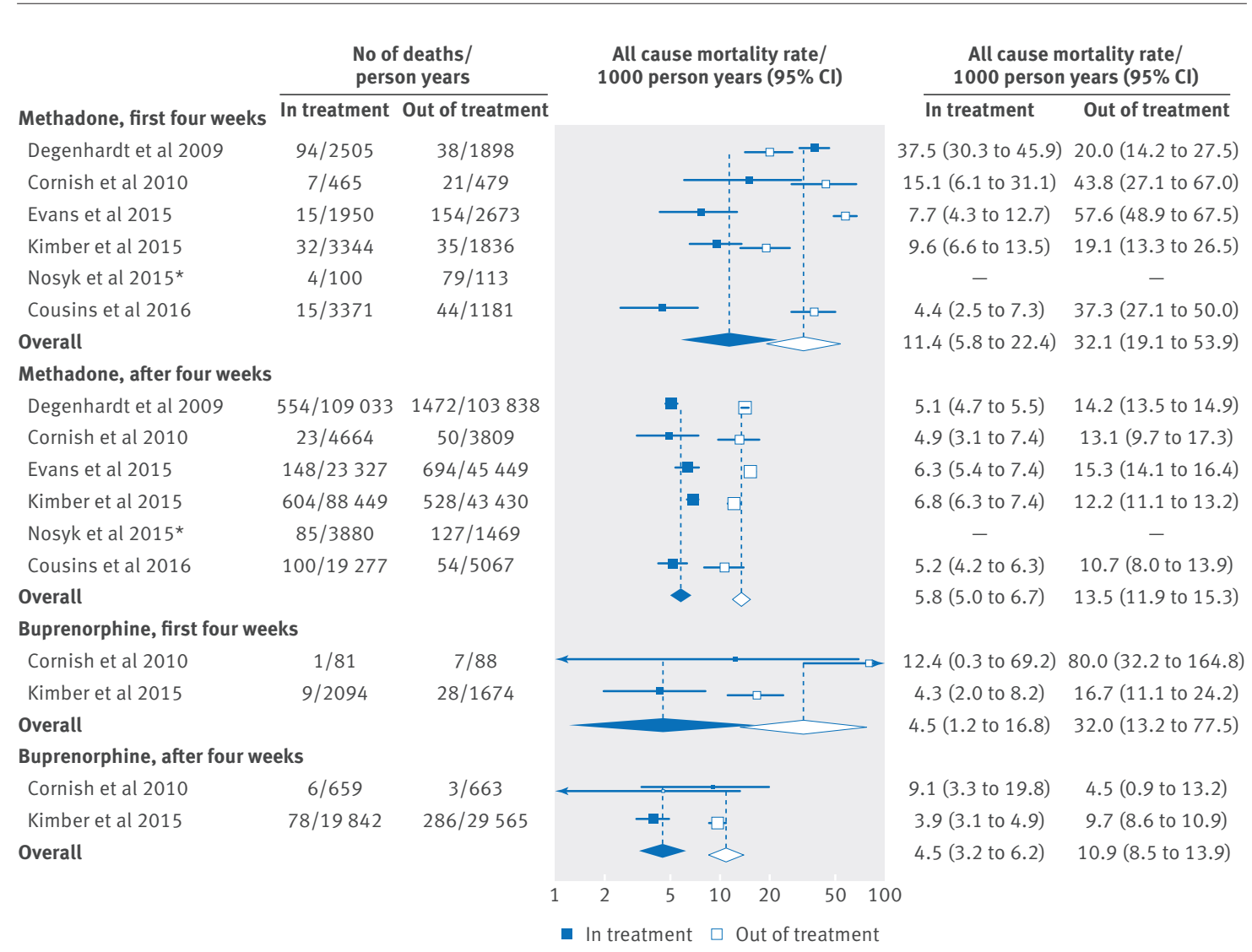

Fig 4 | All cause mortality rates by time interval in and out of opioid substitution treatment with methadone or buprenorphine and pooled all cause mortality rates, 2009-16. Mortality data were disaggregated into first four weeks and remaining follow-up in and out of treatment in all cohort studies except Degenhardt et al, ${ }^{6}$ which reported mortality before and after two weeks of treatment initiation and cessation. High risk cohort of Nosyk et al ${ }^{36}$ (injectors positive for HIV receiving highly active antiretroviral therapy) was excluded from meta-analysis. Area of each square is proportional to study weight in meta-analysis. Horizontal lines represent exact $95 \%$ confidence intervals based on Poisson distribution. Diamonds represent pooled all cause mortality rates before and after four weeks in and out of treatment across methadone or buprenorphine cohorts estimated from multivariate random effects meta-analysis on log transformed rates in four time-by-treatment intervals 

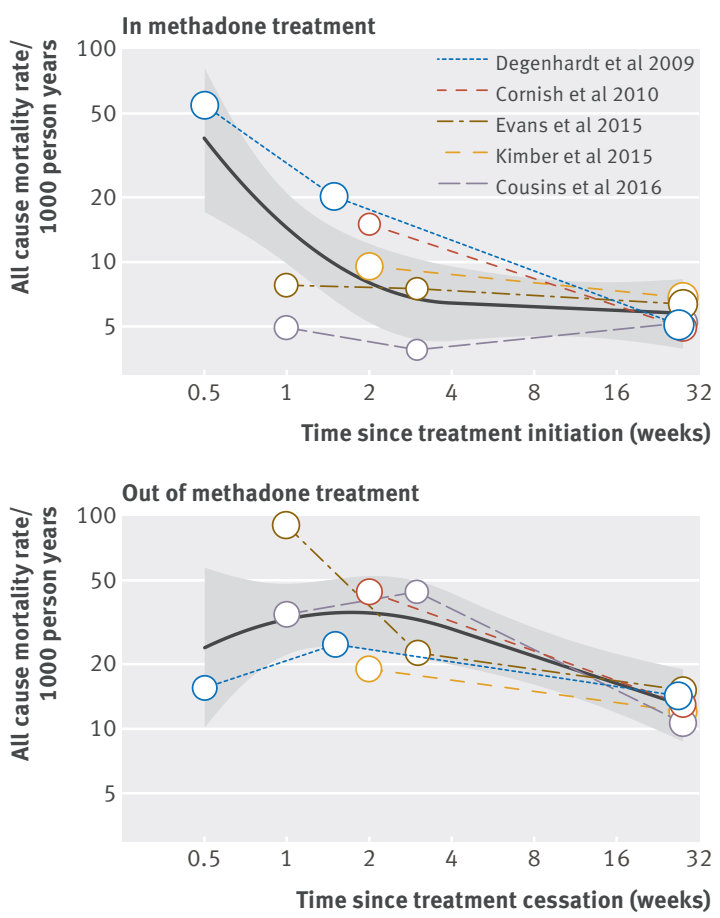

Fig 5 | All cause mortality rates by time since treatment initiation and cessation in methadone cohorts and pooled all cause mortality risk trends, 2009-16. High risk cohort of Nosyk et $\mathrm{al}^{36}$ (injectors positive for HIV receiving highly active antiretroviral therapy) was excluded from meta-regression. Area of each circle is proportional to weight of each time interval in meta-regression. Pooled trends in all cause mortality risk (solid lines) and their $95 \%$ confidence intervals (shaded regions) over time in and out of methadone treatment were estimated from bivariate random effects meta-regression of log transformed rates on quadratic linear spline function of log time with knot at four weeks drug use, comorbidities, prison history, overdose history, patient's preference); characteristics of treatment (such as previous treatment, specialisation of the doctor who controls the treatment, dose, provision characteristics, cointerventions, retention, or drop outs), or the sociopolitical context in which studies have been conducted. For example, the initial prognosis might be better in those given buprenorphine than those given methadone (that is, fewer comorbid problems, less severe opioid dependence), 681061 though this was not clearly found in a recent US study. ${ }^{62}$

The role of such confounding factors has received almost empirical examination. Few details on characteristics of patients or treatments were reported in articles included in this meta-analysis to permit a detailed examination of this potential issue (tables 1 and 2), so it was not possible to assess the possibility of confounding. A sensitivity simulation analysis in the study by Kimber and colleagues, however, suggested that the lower mortality with buprenorphine than with methadone during first four weeks of treatment was unlikely to be caused by unmeasured confounding. ${ }^{10}$

\section{Strengths and limitations}

This meta-analysis synthesised evidence from cohort studies published until 2016 on risk of mortality in people who are dependent on opioids during and after opioid substitution treatment, separately for buprenorphine and methadone. Mortality changes over time (1st-32th week) during and after methadone are carefully quantified for the first time.

The published studies, however, had several methodological shortcomings. Firstly, in the included

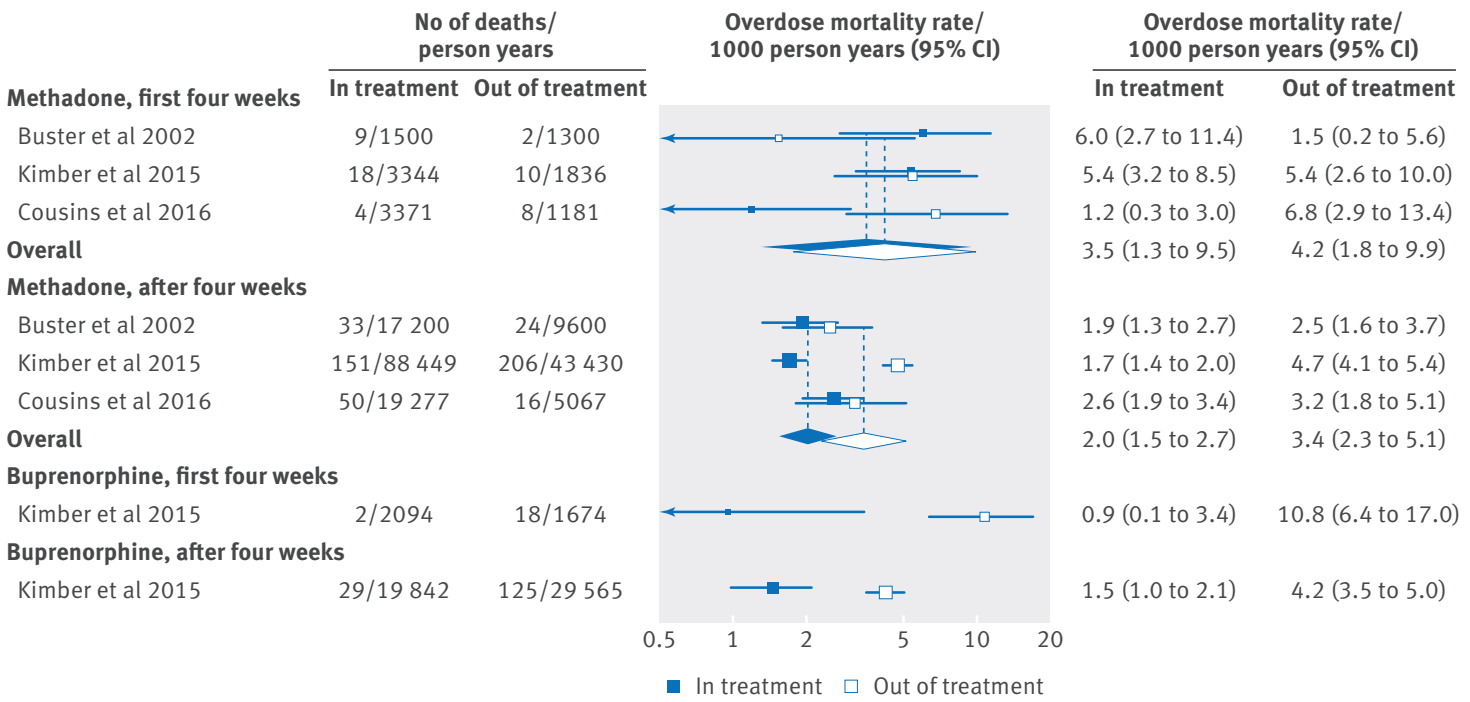

Fig 6 | Overdose mortality rates by time interval in and out of opioid substitution treatment with methadone or buprenorphine and pooled overdose mortality rates, 2002-16. Mortality data were disaggregated into first four weeks and remaining follow-up in and out of treatment in all cohort studies except Buster et al, ${ }^{7}$ which reported mortality before and after two weeks of treatment initiation and cessation. Area of each square is proportional to study weight in metaanalysis. Horizontal lines represent exact $95 \%$ confidence intervals based on Poisson distribution. Diamonds represent pooled overdose mortality rates before and after four weeks in and out of treatment across methadone cohorts estimated from multivariate random-effects meta-analysis on log transformed rates in four time-by-treatment intervals 
observational studies, the same patients are compared throughout follow-up periods in and out of opioid substitution treatment, but these patients leave and re-enter treatment in a non-random way and hence there could still be a large potential for confounding in comparisons of crude mortality risk in and out of treatment. In some studies with diverse information on patient demographics, severity of drug use, and comorbidities measured mostly at baseline (table 3), adjustment for these potential confounders resulted in similar or even increased out-to-in mortality rate ratios, which merely reflects that the above conditions tended to be less prevalent at baseline among patients who later left or were discharged from treatment than those who remained in treatment. Nevertheless, to obtain an unbiased estimate of the preventive effect of opioid substitution treatment on all cause and overdose mortality, these and other relevant confounders should be measured every time a patient leaves and resumes treatment. Time varying data can then be used to adjust for confounder history in standard regression models or, even better, to construct inverse probability of treatment weights for marginal structural models, ${ }^{63}$ which properly control for time varying confounders for later treatment that are themselves affected by previous treatment, such as severity of drug use and comorbidity. Also, differential loss to follow-up is a concern in the meta-analysed cohorts as dropouts are more likely to occur in patients at higher risk of death during periods out of treatment, thus inducing a selection bias in crude comparisons of out-to-in mortality risk because of informative censoring. Marginal structural models with inverse probability of censoring weights can also be used to correct for differential loss to follow-up, ${ }^{63}$ provided that common predictors of death and censoring are measured over time. In summary, individual cohorts are prone to confounding and selection bias because of differential loss to follow-up, but it is hard to assess with the available information the amount and direction of bias projected onto the pooled mortality rate differences and rate ratios between periods in and out of treatment obtained in this meta-analysis.

Secondly, studies were conducted in high income countries, with the follow-up often spread over many calendar years (1965-2010 for methadone and 1990-2010 for buprenorphine) and a highly variable average length of follow-up (ranges 1.3-15.8 years and 1.1-4.5 years, respectively). Studies in low and middle income countries are especially needed.

Thirdly, by design, we have not captured overdose mortality when opioid substitution treatment was obtained on illicit drug markets. ${ }^{4064}$ Fourthly, a delayed record of treatment cessation could have led to some deaths that occurred out of treatment being incorrectly classified as occurring in treatment ${ }^{40}$; if so, this makes our estimated impact of opioid substitution treatment on mortality a conservative one. There could similarly be misclassification between overdose and non-overdose deaths because of inconsistencies in definition of overdose and codification of causes of deaths (for instance, criteria for separating overdose death from intentional poisoning or sudden cardiac death) ${ }^{4065}$; again, these misclassifications would tend to underestimate deaths from overdose.

\section{Implications for policy and practice}

In many countries the number of deaths attributable to opioid use is substantial ${ }^{6667}$ and increasing in some such as the US, where deaths from opioid overdose now outnumber gun related deaths in the country. ${ }^{68}$ Overdoses are preventable causes of death warranting wide implementation of preventive interventions. Our review has provided evidence that methadone and probably buprenorphine reduce mortality among people dependent on opioids, making it imperative that further research confirms this using study designs that consider a wide range of confounding variables to increase confidence that these effects are causal.

It must be borne in mind that there is strong evidence of the efficacy of methadone and buprenorphine on a range of clinical outcomes, and these medicines are listed as WHO essential medicines; the findings we have presented here provide additional imperatives for their widespread availability. Despite this, coverage of opioid substitution treatment is low in many countries worldwide. ${ }^{69}$ Policymakers, clinicians, and those responsible for drug treatment systems should work to ensure the availability of such treatment, remove access barriers, and promote engagement. This means the strategic development of services from a public health perspective, which could reduce the social harm associated with opioid use.

Our review suggests that some precautions should be taken during and after opioid substitution treatment to increase safety. Firstly, careful clinical assessment of opioid tolerance before onset of treatment to establish a safe induction dose seems warranted. Secondly, monitoring during the induction period is important, especially for methadone, with clinicians considering adjusting opioid doses, monitoring mental and somatic problems, and preventing the use of opioids obtained on the illicit drug market. ${ }^{64}$ In addition, education of patients about the risk of overdose risk and use of "take home" naloxone 7071 is warranted. Buprenorphine induction followed by transition to methadone might also be considered..$^{1072}$ Retention in opioid substitution treatment reduces risk of exposure to mortality after cessation and also to re-exposure to mortality risk during induction onto methadone, so efforts to improve retention are important as a strategy to reduce mortality. More generally, establishment of mechanisms for information and coordination between healthcare, social and legal services, and patient counselling while in treatment, in addition to more specific overdose prevention programmes such as naloxone distribution, should be considered.

Finally, there is a pressing need for new studies on the comparative effectiveness of methadone and buprenorphine in reducing mortality. New randomised controlled trials are highly unlikely: they would be prohibitively expensive and difficult to 
implement. A more pragmatic solution is to conduct cohort studies in multiple countries, including both methadone and buprenorphine patients, with heterogeneous characteristics, and sufficient information on potential confounders. These studies could focus on the relation between time in treatment and risks after interruption of treatment to identify a minimum requirement for treatment duration to observe reductions in mortality risk.

Contributors: LS, GB, MJB, BII, MF, and RP-B were involved in the conception and design of the review. LS, GB, and BII developed the search strategy and performed study selection. LS and RP-B extracted data from included studies. LS, GB, and RP-B were involved in the data analysis. LS, GB, MJB, LD, LW, MF, and RP-B were involved in the interpretation and discussion of results. LS, GB, MJB, and RP-B drafted the manuscript, and BII, LD, LW, and MF contributed to the drafting of the review. All authors approved the final version of the article. RP-B is the guarantor.

Funding: This work was partially supported by the ISCIII Network on Addictive Disorders (Networks for Cooperative Research in Health from the Carlos III Institute of Health) (grant No RD16/0017/0013 and RD12/0028/0018) and by the EMCDDA in the context of the activities related to identification, promotion, and monitor of best practices.

Competing interests: All authors have completed the ICMJE uniform disclosure form at http://www.icmje.org/coi_disclosure.pdf and declare: LD has received grants from Reckitt Benckiser/Indivior and grants from Mundipharma outside the submitted work. No further support from any organisation for the submitted work; no other financial relationships with any organisation that might have an interest in the submitted work in the previous three years; no other relationships or activities that could appear to have influenced the submitted work

Ethical approval: Not required.

Transparency: The lead author affirms that the manuscript is an honest, accurate, and transparent account of the study being reported; that no important aspects of the study have been omitted; and that any discrepancies from the study as planned have been disclosed.

Data sharing: No additional data available.

This is an Open Access article distributed in accordance with the Creative Commons Attribution Non Commercial (CC BY-NC 4.0) license, which permits others to distribute, remix, adapt, build upon this work non-commercially, and license their derivative works on different terms, provided the original work is properly cited and the use is non-commercial. See: http://creativecommons.org/licenses/ by-nc/4.0/.

1 Degenhardt L, Charlson F, Mathers B, et al. The global epidemiology and burden of opioid dependence: results from the global burden of disease 2010 study. Addiction 2014;109:1320-33. doi:10.1111/add.12551.

2 Mattick RP, Breen C, Kimber J, Davoli M. Methadone maintenance therapy versus no opioid replacement therapy for opioid dependence. Cochrane Database Syst Rev 2009:(3):CD002209.

3 Mattick RP, Breen C, Kimber I, Davoli M. Buprenorphine maintenance versus placebo or methadone maintenance for opioid dependence. Cochrane Database Syst Rev 2014;2:CD002207.

4 Lawrinson P, Ali R, Buavirat A, et al. Key findings from the WHO collaborative study on substitution therapy for opioid dependence and HIV/AIDS. Addiction 2008;103:1484-92. doi:101111/j.1360-0443.2008.02249x

5 Ward J, Hall W, Mattick RP. Role of maintenance treatment in opioid dependence. Lancet 1999;353:221-6. doi:10.1016/ S0140-6736(98)05356-2.

6 Degenhardt L, Randall D, Hall W, Law M, Butler T, Burns L. Mortality among clients of a state-wide opioid pharmacotherapy program over 20 years: risk factors and lives saved. Drug Alcohol Depend 2009:105:9-15. doi:10.1016/j.drugalcdep.2009.05.021.

7 Buster MC, van Brussel GH, van den Brink W. An increase in overdose mortality during the first 2 weeks after entering or re-entering methadone treatment in Amsterdam. Addiction 2002;97:993-1001. doi:10.1046/j.1360-0443.2002.00179x.

8 Cornish R, Macleod J, Strang J, Vickerman P, Hickman M. Risk of death during and after opiate substitution treatment in primary care: prospective observational study in UK General Practice Research Database. BMJ 2010;341:c5475. doi:10.1136/bmj.c5475.

9 Evans E, Li L, Min J, et al. Mortality among individuals accessing pharmacological treatment for opioid dependence in California 2006-10. Addiction 2015:110:996-1005. doi:10.1111/add.12863.
10 Kimber J, Larney S, Hickman M, Randall D, Degenhardt L. Mortality risk of opioid substitution therapy with methadone versus buprenorphine: a retrospective cohort study. Lancet Psychiatry 2015;2:901-8. doi:10.1016/S2215-0366(15)00366-1.

11 Degenhardt L, Bucello C, Mathers B, et al. Mortality among regular or dependent users of heroin and other opioids: a systematic review and meta-analysis of cohort studies. Addiction 2011;106:32-51. doi:10.1111/j.1360-0443.2010.03140.x

12 Hickman M, Macleod J, Degenhardt L. Commentary on Cousins et al. (2016): Accumulating evidence on risk of mortality on and off opioid substitution treatment. Addiction 2016;111:83-4. doi:10.1111/ add.13185.

13 Moher D, Liberati A, TetzlaffJ, Altman DG. PRISMA Group. Preferred reporting items for systematic reviews and meta-analyses: the PRISMA statement. BMJ 2009;339:b2535. doi:10.1136/bmj.b2535.

14 Scottish Intercollegiate Guidelines Network (SIGN). SIGN 50: a guidelines developers' handbook. 2014. Edinburgh, Scottish Intercollegiate Guidelines Network (SIGN).

15 Degenhardt L, Calabria B, Nelson P, et al, and Global Burden of Disease Mental Disorders and Illicit Drug Use Expert group. Methodology used in a systematic review of evidence on the prevalence of amphetamine use and dependence. 2009. Sydney, National Drug and Alcohol Research Centre, University of NSW. Illicit drugs discussion paper No.13.

16 Wells G, Shea B. O'Connell D, et al. The Newcastle-Ottawa Scale (NOS) for assessing the quality of non-randomised studies in meta-analyses. In: 3rd Symposium on Systematic Reviews: Beyondthe Basics. Improving Quality and Impact, Oxford, UK. 2013.

17 Berkey CS, Hoaglin DC, Antczak-Bouckoms A, Mosteller F, Colditz GA. Meta-analysis of multiple outcomes by regression with random effects. Stat Med 1998;17:2537-50. doi:10.1002 (SICI)1097-0258(19981130)17:22<2537::AID-SIM953>3.0.CO;2-C.

18 Gasparrini A, Armstrong B, Kenward MG. Multivariate meta-analysis for non-linear and other multi-parameter associations. Stat Med 2012;31:3821-39. doi:10.1002/sim.5471

19 Jackson D, Riley R, White IR. Multivariate meta-analysis: potential and promise. StatMed 2011;30:2481-98. doi:10.1002/sim.4247.

20 Greenland S. Dose-response and trend analysis in epidemiology: alternatives to categorical analysis. Epidemiology 1995;6:356-65. doi:10.1097/00001648-199507000-00005.

21 Jackson D, White IR, Riley RD. Quantifying the impact of betweenstudy heterogeneity in multivariate meta-analyses. Stat Med 2012;31:3805-20. doi:10.1002/sim.5453.

22 Egger M, Davey Smith G, Schneider M, Minder C. Bias in meta-analysis detected by a simple, graphical test. BMJ 1997;315:629-34. doi:10.1136/bmj.315.7109.629.

23 Thompson SG, Sharp SJ. Explaining heterogeneity in meta-analysis: a comparison of methods. Stat Med 1999:18:2693-708. doi:10.1002/ (SICI)1097-0258(19991030)18:20<2693::AID-SIM235>3.0.CO;2-V.

24 Gearing FR, Schweitzer MD. An epidemiologic evaluation of long-term methadone maintenance treatment for heroin addiction. Am J Epidemiol 1974;100:101-12. doi:10.1093/oxfordjournals.aje. a112012.

25 Cushman PJr. Ten years of methadone maintenance treatment: some clinical observations. Am I Drug Alcohol Abuse 1977;4:543-53. doi:10.3109/00952997709007010.

26 Grönbladh L, Ohlund LS, Gunne LM. Mortality in heroin addiction: impact of methadone treatment. Acta Psychiatr Scand 1990;82:223-7. doi:10.1111/j.1600-0447.1990.tb03057.x

27 Caplehorn JR, Dalton MS, Cluff MC, Petrenas AM. Retention in methadone maintenance and heroin addicts' risk of death. Addiction 1994;89:203-9. doi:10.1111/j.1360-0443.1994.tb00879.x.

28 Caplehorn JR, Dalton MS, Haldar F, Petrenas AM, Nisbet JG Methadone maintenance and addicts' risk of fatal heroin overdose. Subst Use Misuse 1996;31:177-96. doi:10.3109/10826089609045806

29 Fugelstad A, Rajs J, Böttiger M, Gerhardsson de Verdier M. Mortality among HIV-infected intravenous drug addicts in Stockholm in relation to methadone treatment. Addiction 1995;90:711-6. doi:10.1111/j.1360-0443.1995.tb02209.x.

30 Fugelstad A, Agren G, Romelsjö A. Changes in mortality, arrests, and hospitalizations in nonvoluntarily treated heroin addicts in relation to methadone treatment. Subst Use Misuse 1998;33:2803-17. doi:10.3109/10826089809059352.

31 Scherbaum N, Specka M. Hauptmann G, Gastpar M. [Does maintenance treatment reduce the mortality rate of opioid addicts?]. Fortschr Neurol Psychiatr 2002;70:455-61 doi:10.1055/s-2002-33758

32 Davoli M, Bargagli AM, Perucci CA, et al. VEdeTTE Study Group. Risk of fatal overdose during and after specialist drug treatment: the VEdeTTE study, a national multi-site prospective cohort study. Addiction 2007:102:1954-9. doi:10.1111/j.1360-0443.2007.02025.x

33 Fugelstad A, Stenbacka M, Leifman A, Nylander M, Thiblin I. Methadone maintenance treatment: the balance between life-saving treatment and fatal poisonings. Addiction 2007;102:406-12. doi:10.1111/i.1360-0443.2006.01714.x 
34 Clausen T, Anchersen K, Waal H. Mortality prior to, during and after opioid maintenance treatment (OMT): a national prospective cross-registry study. Drug Alcohol Depend 2008:94:151-7. doi:10.1016/j.drugalcdep.2007.11.003

35 Peles E, Schreiber S, Adelson M. 15-Year survival and retention of patients in a general hospital-affiliated methadone maintenance treatment (MMT) center in Israel. Drug Alcohol Depend 2010;107:1418. doi:10.1016/j.drugalcdep.2009.09.013.

36 Nosyk B, Min JE, Evans E, et al. The Effects of Opioid Substitution Treatment and Highly Active Antiretroviral Therapy on the Cause-Specific Risk of Mortality Among HIV-Positive People Who Inject Drugs. Clin Infect Dis 2015;61:1157-65. doi:10.1093/cid/civ476.

37 Cousins G, Boland F, Courtney B, Barry J, Lyons S, Fahey T. Risk of mortality on and off methadone substitution treatment in primary care: a national cohort study. Addiction 2016;111:73-82. doi:10.1111/ add.13087.

38 Reece AS. Favorable mortality profile of naltrexone implants for opiate addiction. J Addict Dis 2010;29:30-50. doi:10.1080/10550880903435988.

39 Auriacombe M. Franques P. Tignol J. Deaths attributable to methadone vs buprenorphine in France. JAMA 2001;285:45. doi:10.1001/ jama.285.1.39.

40 Bell J, Trinh L, Butler B, Randall D, Rubin G. Comparing retention in treatment and mortality in people after initial entry to methadone and buprenorphine treatment. Addiction 2009;104:1193-200. doi:10.1111/j.1360-0443.2009.02627.x.

41 Kleber HD. Pharmacologic treatments for opioid dependence: detoxification and maintenance options. Dialogues Clin Neurosci 2007;9:455-70.

42 Pirnay S, Borron SW, Giudicelli CP, Tourneau J, Baud FJ, Ricordel I. A critical review of the causes of death among post-mortem toxicological investigations: analysis of 34 buprenorphine-associated and 35 methadone-associated deaths. Addiction 2004;99:978-88. doi:10.1111/i.1360-0443.2004.00790.x.

43 Soyka M, Apelt SM, Lieb M, Wittchen HU. One-year mortality rates of patients receiving methadone and buprenorphine maintenance therapy: a nationally representative cohort study in 2694 patients. I Clin Psychopharmacol 2006;26:657-60. doi:10.1097/01. jcp.0000245561.99036.49.

44 Thomas CP, Fullerton CA, Kim M, et al. Medication-assisted treatment with buprenorphine: assessing the evidence. Psychiatr Serv 2014;65:158-70. doi:10.1176/appi.ps.201300256

45 Brugal MT, Domingo-Salvany A, Puig R, Barrio G, García de Olalla P, de la Fuente L. Evaluating the impact of methadone maintenance programmes on mortality due to overdose and aids in a cohort of heroin users in Spain. Addiction 2005;100:981-9. doi:10.1111/j.1360-0443.2005.01089.x

46 Bell J, Burrell T, Indig D, Gilmour S. Cycling in and out of treatment; participation in methadone treatment in NSW, 1990-2002. Drug Alcohol Depend 2006;81:55-61. doi:10.1016/j. drugalcdep.2005.05.010

47 Cousins G, Teljeur C, Motterlini N, McCowan C, Dimitrov BD, Fahey T. Risk of drug-related mortality during periods of transition in methadone maintenance treatment: a cohort study. J Subst Abuse Treat 2011:41:252-60. doi:10.1016/i.jsat.2011.05.001.

48 Hser YI, Evans E, Grella C, Ling W, Anglin D. Long-term course of opioid addiction. Harv Rev Psychiatry 2015;23:76-89. doi:10.1097/ HRP.0000000000000052.

49 Nordt C, Vogel M, Dürsteler KM, Stohler R, Herdener M. A comprehensive model of treatment participation in chronic disease allowed prediction of opioid substitution treatment participation in Zurich, 1992-2012. J Clin Epidemiol 2015;68:1346-54. doi:10.1016/j. jclinepi.2015.05.002.

50 Teesson M, Marel C, Darke S, et al. Long-term mortality, remission, criminality and psychiatric comorbidity of heroin dependence: 11-year findings from the Australian Treatment Outcome Study. Addiction 2015;110:986-93. doi:10.1111/add.12860.

51 Eap CB, Buclin T, Baumann P. Interindividual variability of the clinical pharmacokinetics of methadone: implications for the treatment of opioid dependence. Clin Pharmacokinet 2002;41:1153-93. doi:10.2165/00003088-200241140-00003.

52 Jolley CJ, Bell J, Rafferty GF, Moxham J, Strang J. Understanding heroin overdose: a study of the acute respiratory depressant effects of injected pharmaceutical heroin. PLoS One 2015;10:e0140995. doi:10.1371/journal.pone.0140995.

53 McCowan C, Kidd B, Fahey T. Factors associated with mortality in Scottish patients receiving methadone in primary care: retrospective cohort study. BMJ 2009;338:b2225. doi:10.1136/bmj.b2225.
54 Yokell MA, Zaller ND, Green TC, Rich JD. Buprenorphine and buprenorphine/naloxone diversion, misuse, and illicit use: an international review. Curr Drug Abuse Rev 2011;4:28-41. doi:10.2174/1874473711104010028.

55 European Monitoring Centre for Drugs and Drug Addiction (EMCDDA). Mortality realted to drug use in Europe: Public Health Implications. EMCDDA (European Monitoring Centre for Drugs and Drug Addiction).Publications Office of the European Union. Selected Issues, 2011.

56 White JM, Irvine RJ. Mechanisms of fatal opioid overdose. Addiction 1999;94:961-72. doi:10.1046/j.1360-0443.1999.9479612.x.

57 Binswanger IA, Blatchford PJ, Mueller SR, Stern MF. Mortality after prison release: opioid overdose and other causes of death, risk factors, and time trends from 1999 to 2009. Ann Intern Med 2013; 159:592-600 doi:10.7326/0003-4819-159-9-201311050-00005.

58 Merrall EL, Kariminia A, Binswanger IA, et al. Meta-analysis of drug-related deaths soon after release from prison. Addiction 2010;105:1545-54. doi:10.1111/j.1360-0443.2010.02990.x.

59 Merrall EL, Bird SM, Hutchinson SJ. A record-linkage study of drug-related death and suicide after hospital discharge among drug-treatment clients in Scotland, 1996-2006. Addiction 2013;108:377-84. doi:10.1111/j.1360-0443.2012.04066.x.

60 Bell JR, Butler B, Lawrance A, Batey R, Salmelainen P. Comparing overdose mortality associated with methadone and buprenorphine treatment. Drug Alcohol Depend 2009;104:73-7. doi:10.1016/j. drugalcdep.2009.03.020

61 Brogly SB, Saia KA, Walley AY, Du HM, Sebastiani P. Prenatal buprenorphine versus methadone exposure and neonatal outcomes: systematic review and meta-analysis. Am J Epidemiol 2014;180:67386. doi:10.1093/aje/kwu190.

62 Manhapra A, Quinones L, Rosenheck R. Characteristics of veterans receiving buprenorphine vs. methadone for opioid use disorder nationally in the Veterans Health Administration. Drug Alcohol Depend 2016;160:82-9. doi:10.1016/j.drugalcdep.2015.12.035.

63 Robins JM, Hernán MA, Brumback B. Marginal structural models and causal inference in epidemiology. Epidemiology 2000;11:550-60. doi:10.1097/00001648-200009000-00011.

64 Rhodes T, Hedrich D. Strategies to prevent diversion of opioid substitution treatment medications. European Monitoring Centre for Drugs and Drug Addiction, 2016.

65 Gossop M, Stewart D, Treacy S, Marsden J. A prospective study of mortality among drug misusers during a 4-year period after seeking treatment. Addiction 2002;97:39-47. doi:10.1046/j.1360-0443.2002.00079.x

66 European Monitoring Centre for Drugs and Drug Addiction (EMCDDA). European Drug Report, 2015. Trends and developments. EMCDDA (European Monitoring Centre for Drugs and Drug Addiction).Publications Office of the European Union. EMCDDA Papers, 2015.

67 United Nations Office on Drugs and Crime. World Drug Report 2015. 2015. United Nations publication, Sales No. E. 14.XI.7.

68 Rudd RA, Seth P, David F, Scholl L. Increases in Drug and OpioidInvolved Overdose Deaths - United States, 2010-2015. MMWR Morb Mortal Wkly Rep 2016;65:1445-52. doi:10.15585/mmwr. $\mathrm{mm} 655051 \mathrm{e} 1$

69 Mathers BM, Degenhardt L, Ali H, et al. 2009 Reference Group to the UN on HIV and Injecting Drug Use. HIV prevention, treatment, and care services for people who inject drugs: a systematic review of global, regional, and national coverage. Lancet 2010;375:1014-28. doi:10.1016/S0140-6736(10)60232-2.

70 European Monitoring Centre for Drugs and Drug Addiction (EMCDDA) Preventing overdose deaths in Europe. 2015. Lisbon, European Monitoring Centre for Drugs and Drug Addiction (EMCDDA).

71 Zador D, Sunjic S. Deaths in methadone maintenance treatment in New South Wales, Australia 1990-1995. Addiction 2000;95:77-84. doi:10.1046/j.1360-0443.2000.951778.x

72 Kakko J, Grönbladh L, Svanborg KD, et al. A stepped care strategy using buprenorphine and methadone versus conventional methadone maintenance in heroin dependence: a randomized controlled trial. Am J Psychiatry 2007;164:797-803. doi:10.1176/ ajp.2007.164.5.797.
Appendix 1: Searches
Appendix 2: Supplementary tables A and B Appendix 3: Quality score 